\title{
A prospective cohort study of patients with non-squamous non-small cell lung cancer treated with bevacizumab
}

\author{
KATSUHIKO NAOKI ${ }^{1-3^{*}}$, YUICHIRO TAKEDA ${ }^{3,4^{*}}$, KENZO SOEJIMA $^{2,3}$, DAISUKE ARAI ${ }^{2,3}$, \\ GO NAKA $^{3,4}$, SEISUKE NAGASE ${ }^{3,5}$, KEN ARIMURA $^{3,6}$, TOSHINORI KANEMURA ${ }^{3,6}$, \\ TATSUO OHHIRA $^{3,5}$ and NORIHIKO IKEDA ${ }^{3,5}$ \\ ${ }^{1}$ Cancer Center, ${ }^{2}$ Division of Pulmonary Medicine, Department of Medicine, Keio University School of Medicine; \\ ${ }^{3}$ Shinjuku Thoracic Oncology Group, Tokyo 160-8582; ${ }^{4}$ Department of Respiratory Medicine, National Center \\ for Global Health and Medicine, Tokyo $162-8655 ;{ }^{5}$ Department of Surgery I, Tokyo Medical University, \\ Tokyo 160-0023; ${ }^{6}$ First Department of Medicine and Department of Chemotherapy and Palliative Care, \\ Tokyo Women's Medical University, Tokyo 162-8666, Japan
}

Received May 6, 2016; Accepted January 19, 2017

DOI: 10.3892/ol.2017.5796

\begin{abstract}
First-line chemotherapy regimens that include bevacizumab (Bev) have been hypothesized to improve outcomes in patients with advanced non-squamous non-small cell lung cancer (non-sq NSCLC). Although approved to treat NSCLC in 2009, insufficient data exist on the clinical uses of Bev in Japan. The present study prospectively evaluated the efficacy and safety of Bev-containing combination chemotherapy. Eligible patients exhibited histologically or cytologically documented advanced or recurrent non-sq NSCLC. Patients were administered $15 \mathrm{mg} / \mathrm{kg}$ Bev with standard chemotherapy followed by maintenance Bev. The primary endpoints were progression-free survival (PFS) and safety. A total of 102 patients with non-sq NSCLC were enrolled, $44.1 \%$ of whose tumor carried epidermal growth factor receptor $(E G F R)$ mutations. The overall response rate to the intervention was $44.1 \%$, and the median PFS was 8.3 months [95\% confidence interval $(\mathrm{CI})=6.4-10.2$ months]. The median overall survival was 26.3 months (95\% CI=22.2-30.4 months). The incidence of Bev-associated severe adverse events was similar to those in previous trials, excluding a grade 3-4 hypertension rate of $30.4 \%$ in the present study. Multivariate analysis revealed that a higher TNM classification of malignant tumor staging-T factor, adjusted hazard ratio $(\mathrm{HR})=1.33(95 \% \mathrm{CI}=1.10-1.61)$, and poor performance status [adjusted $\mathrm{HR}=1.63(1.02-2.60)]$ were
\end{abstract}

Correspondence to: Dr Katsuhiko Naoki, Cancer Center, Keio University School of Medicine, 35 Shinanomachi, Shinjuku-ku, Tokyo 160-8582, Japan

E-mail: naoki@z5.keio.jp

*Contributed equally

Key words: bevacizumab, non-small-cell lung cancer, prospective, cohort study, endothelial growth factor receptor associated with significantly shorter PFS, whilst the EGFR exon 19 deletion was significantly associated with prolonged PFS [adjusted HR=0.47 (0.25-0.87)]. Bev-containing chemotherapy was safe and effective for patients with non-sq NSCLC in clinical settings in Japan. The EGFR exon 19 deletion was suggested as a positive predictive factor for the efficacy of Bev-containing chemotherapy.

\section{Introduction}

In several clinical trials, first-line combination chemotherapies containing bevacizumab (Bev) were revealed to improve clinical outcomes in patients with advanced non-squamous non-small cell lung cancer (non-sq NSCLC) (1-3). Sandler et al (3) reported significant survival benefits, such as overall survival (OS) of $>1$ year (12.3 months), with addition of Bev to paclitaxel plus carboplatin in the treatment of non-sq NSCLC. Although Bev was approved for NSCLC in 2009 in Japan, there are insufficient data regarding the efficacy, toxicity and predictive markers for Bev treatment. The present study evaluated the efficacy and safety of Bev-containing combination chemotherapy in patients with non-sq NSCLC in clinical settings in Japan. Landmark survival analysis, or disease control at 8 weeks, was reported to be a more powerful predictor of subsequent survival compared with the traditional tumor response rate in advanced NSCLC (4). This may provide an early assessment of subsequent outcome. Since treatment with bevacizumab occasionally results in cavitary lesion without tumor shrinkage (5), stable disease may also be important for understanding drug efficacy. Therefore, landmark analysis was utilized in the present study. In addition, the identification of predictive markers for Bev-containing chemotherapy efficacy was attempted. The mutation of epidermal growth factor receptor $(E G F R)$ is a key factor in predicting the response and survival rate following EGFR-tyrosine kinase inhibitor (EGFR-TKI) treatment $(6,7)$, but no data have demonstrated the importance of EGFR mutations in predicting the effect of Bev treatment. In the present study, multivariate analysis using Cox's regression model 
revealed that specific $E G F R$ mutations are predictive markers for Bev treatment efficacy.

\section{Patients and methods}

Patient eligibility. Patients scheduled for Bev treatment between August 2010 to July 2012 were prospectively enrolled in the present study. Eligible patients had histologically or cytologically confirmed inoperable advanced, stage IIIB-IV, or recurrent non-sq NSCLC, an Eastern Cooperative Oncology Group (ECOG) performance status (PS) of 0-2, and adequate organ function for cytotoxic chemotherapy. The main exclusion criteria were diagnoses of squamous cell carcinoma or symptomatic brain metastasis, although patients with controlled brain metastases were eligible, surgery or surgical biopsy within 4 weeks, a history of significant hemoptysis, of $>2.5 \mathrm{ml}$ per episode, coagulation treatment, bleeding tendency, active concomitant malignancy, presence of significant comorbidities such as uncontrolled hypertension, interstitial pneumonia, active gastrointestinal ulcer, angina pectoris, pregnancy or lactation or other factors as judged by a medical oncologist. Protocol-specified demographics, disease characteristics including EGFR mutation status and patient medical history were collected at baseline. Patients were treated at four major hospitals participating in the Shinjuku Thoracic Oncology Group (STOG), Keio University School of Medicine (Tokyo, Japan), National Center for Global Health and Medicine (Tokyo, Japan), Tokyo Medical University (Tokyo, Japan), Tokyo Women's Medical University (Tokyo, Japan). The study protocol was approved by the institutional review board at each institution. All patients provided written informed consent prior to inclusion in the present study.

Study design and treatment. The primary endpoints were progression-free survival (PFS) and safety. The secondary endpoints were the response rate (RR), time to response and landmark survival (4). Patients received $15 \mathrm{mg} / \mathrm{kg}$ Bev every 3 weeks in conjunction with the chemotherapy prescribed by attending physicians, followed by $15 \mathrm{mg} / \mathrm{kg}$ Bev every 3 weeks with or without chemotherapy as maintenance. Any line of chemotherapy was permitted. Bev-containing regimens were as follows: Carboplatin (CBDCA) + pemetrexed (PEM); cisplatin $(\mathrm{CDDP})+$ PEM; CBDCA + paclitaxel (PTX); PEM; docetaxel (DTX); CBDCA + DTX; and CBDCA + PEM + erlotinib $(n=41$, $n=21, n=18, n=9, n=9, n=3$ and $n=1$, respectively). All chemotherapy regimens contained either PEM $(n=72)$ or a taxane (DTX or PTX; $\mathrm{n}=30$ ). Treatment was continued until tumor progression or the patient experienced unacceptable toxicities, such as grade 2 or severe hemoptysis, grade 3 or severe bleeding, or by the decision of attending physician.

Evaluation. Tumor response was assessed according to the Response Evaluation Criteria in Solid Tumors version 1.1 (8). Tumors were assessed at baseline by computed tomography, magnetic resonance imaging, bone scintigraphy and/or fluorodeoxyglucose positron emission tomography. During treatment, a radiographic evaluation was performed subsequent to at least every two courses of treatment and/or at the time of suspected disease progression. To confirm response, a partial response (PR) or complete response (CR), radiographic evaluations were recommended 4 weeks subsequent to the original evaluations. The disease control rate (DCR) was defined as $[\mathrm{CR}+\mathrm{PR}+$ stable disease (SD)]/total patients. Toxicities were graded according to the National Cancer Institute Common Toxicity Criteria version 4.0 (9).

Statistical analysis. The primary endpoints were PFS and safety. Using the 13-week PFS rates of $80.1 \%$ in the chemotherapy plus Bev arm and $67.7 \%$ in the chemotherapy-alone arm from the ECOG 4599 study (3), the sample size was calculated to achieve a power of $80 \%$ with a two-sided $\alpha$ of 0.05 , and the expected and threshold values for PFS were 6.2 and 4.5 months, respectively. The estimated minimum sample size was 77 , which was calculated by SWOG statistical tools using a one-arm binomial setup. Allowing for a maximum dropout rate of $30 \%$ and considering that the present study was an observational cohort study with a minimum of 12 months of follow-up, 100 patients were sought for enrollment. PFS and OS subsequent to the initiation of the Bev-containing chemotherapy were estimated by the Kaplan-Meier method. PFS was defined as the time from the initiation of Bev therapy to investigator-assessed disease progression or mortality from any cause. OS was defined as the time from the initiation of the Bev therapy to mortality from any cause.OS,PFS and responses were assessed in alleligible patients on an intent-to-treat basis. Patients without an event (progression or mortality) were censored at the last follow-up or data cutoff date, whichever occurred first. Univariate and multivariate analyses were performed using Cox's proportional hazards model to assess the independent effects of patient and disease characteristics on PFS and OS. To avoid possible confounding effects of treatment with EGFR-TKIs prior to accrual of the present study ('pre-treatment with EGFR-TKIs') multivariate analyses of PFS and OS were adjusted for 'pre-treatment with EGFR-TKIs'. Landmark survival analyses (4) of PFS and OS were performed by comparing the patients who achieved PR or SD 8 weeks subsequent to Bev administration. All P-values in the present study are two-sided. $\mathrm{P}<0.05$ was considered to indicate a statistically significant difference. Statistical analysis was conducted using IBM SPSS Statistics version 19.0 software (IBM SPSS, Armonk, NY, USA). The present study is registered with the University Hospital Medical Information Network (UMIN) Clinical Trials Registry (www.umin.ac.jp/ctr) as trial number UMIN000004609.

\section{Results}

Patients characteristics and treatment. Between August 2010 and July 2012, a total of 102 patients were enrolled in the present study, with a data cutoff date of September 30, 2014. The duration of follow-up subsequent to final registration was 14 months. The median follow-up time was 1,021 days (33.6 months). Patient characteristics are summarized in Table I. Histological analysis revealed that 98 patients $(96.1 \%)$ exhibited adenocarcinoma, three patients exhibited NSCLC that was not otherwise specified, and one patient exhibited pleomorphic carcinoma. The majority of the patients were receiving first-line treatment $(\mathrm{n}=57,56 \%)$, but patients receiving second-line $(\mathrm{n}=22,22 \%)$, third-line $(\mathrm{n}=12,12 \%)$ and later lines of treatment, fourth, fifth, sixth and seventh line, $n=5, n=4, n=1$, and $n=1$, respectively, were also included. At the data cutoff date, the median numbers of cycles of Bev administration were 
Table I. Patient characteristics $(n=102)$.

\begin{tabular}{|c|c|c|}
\hline Characteristic & Classification & $\mathrm{n}(\%)$ \\
\hline Age, years & (median, range) & $64(36-85)$ \\
\hline \multirow[t]{2}{*}{ Sex } & Male & $60(58.8)$ \\
\hline & Female & $42(41.2)$ \\
\hline \multirow[t]{2}{*}{ Smoking history } & + & $61(59.8)$ \\
\hline & - & $41(40.2)$ \\
\hline \multirow[t]{3}{*}{ Performance status } & 0 & $66(64.7)$ \\
\hline & 1 & $34(33.3)$ \\
\hline & 2 & $2(2.0)$ \\
\hline \multirow[t]{2}{*}{ Histology } & Adenocarcinoma & $98(96.1)$ \\
\hline & Others & $4 \quad(3.9)$ \\
\hline \multirow[t]{4}{*}{ Stage } & IIIA & $2(2.0)$ \\
\hline & IIIB & $8 \quad(7.8)$ \\
\hline & IV & $77(75.5)$ \\
\hline & Recurrent & $15(14.7)$ \\
\hline \multirow[t]{2}{*}{ Brain metastasis } & + & $11(10.8)$ \\
\hline & - & $91(89.2)$ \\
\hline \multirow[t]{2}{*}{ Platinum combination } & + & $84(82.4)$ \\
\hline & - & $18(17.6)$ \\
\hline \multirow[t]{3}{*}{ Treatment line } & First & $57(55.9)$ \\
\hline & Second & $22(21.6)$ \\
\hline & Third and later & $23(22.5)$ \\
\hline \multirow[t]{3}{*}{ EGFR mutation } & + & $45(44.1)^{\mathrm{a}}$ \\
\hline & - & $55(53.9)$ \\
\hline & Unknown & $2(2.0)$ \\
\hline
\end{tabular}

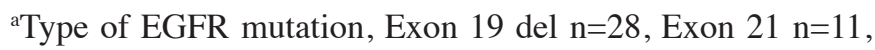
no information $\mathrm{n}=6$.

9,6 , and 7 for the first-, second-, and third- and later lines of treatment, respectively. EGFR mutation status was available for the majority of the patients $(98 \%)$, with mutations identified in $44.1 \%$ of the patients. Amongst the patients with EGFR mutations, mutation types were characterized for 39 patients. The most common type of mutation was an exon 19 deletion ( $n=28$, including one case with T790M), followed by the exon 21 mutations L858R, L858R and T790M, and L861Q $(\mathrm{n}=11$ total; $\mathrm{n}=8 ; \mathrm{n}=2 ; \mathrm{n}=1$, respectively). Amongst the patients with major EGFR mutations, 14 of 28 patients (50\%) with the exon 19 deletion and 8 of 11 patients $(73 \%)$ with the exon 21 mutations received 'pre-treatment with EGFR-TKIs'. In total, 12 of 28 patients (43\%) with the exon 19 deletion and 8 of 11 patients $(73 \%)$ with the exon 21 mutations were treated with EGFR-TKIs subsequent to completion of the present study, that is, 'post-treatment with EGFR-TKIs'. Only five patients with the exon 19 deletion lacked information regarding EGFR-TKI treatment, but all patients with the exon 21 mutations received EGFR-TKIs prior and/or subsequent to the present study.

Toxicity. The toxicity profile is summarized in Table II. The most frequent grade 3 or 4 adverse events were neutropenia and hypertension. Severe grade 3 or 4 hematological toxicities included leukopenia (25.5\%), neutropenia (42.2\%), anemia $(6.9 \%)$, thrombocytopenia $(4.9 \%)$ and febrile neutropenia
Table II. Toxicities observed.

\begin{tabular}{|c|c|c|}
\hline & $\begin{array}{c}\text { All } \\
\mathrm{n}(\%)\end{array}$ & $\begin{array}{c}\text { G3 or more } \\
\mathrm{n}(\%)\end{array}$ \\
\hline Anemia & $58(56.9)$ & $7 \quad(6.9)$ \\
\hline Thrombocytopenia & $47(46.1)$ & $5 \quad(4.9)$ \\
\hline Leukopenia & $69(67.6)$ & $26(25.5)$ \\
\hline Neutropenia & $65(63.7)$ & $43(42.2)$ \\
\hline Febrile neutropenia & $4 \quad(3.9)$ & $4 \quad(3.9)$ \\
\hline Hypoalbuminemia & $42(41.2)$ & $1 \quad(1.0)$ \\
\hline Increased AST & $45(44.1)$ & $3 \quad(2.9)$ \\
\hline Increased ALT & $40(39.2)$ & $2(2.0)$ \\
\hline Increased ALP & $19(18.6)$ & \\
\hline Increased creatinine & $15(14.7)$ & \\
\hline Proteinuria & $49(48.0)$ & $6 \quad(5.9)$ \\
\hline Declining PS & $56(54.9)$ & $10 \quad(9.8)$ \\
\hline Nausea & $42(41.2)$ & $2 \quad(2.0)$ \\
\hline Vomiting & $16(15.7)$ & $1 \quad(1.0)$ \\
\hline Appetite loss & $52(51.0)$ & $2(2.0)$ \\
\hline Diarrhea & $10 \quad(9.8)$ & \\
\hline Constipation & $40(39.2)$ & \\
\hline Mucositis oral & $11(10.8)$ & \\
\hline Fatigue & $27(26.5)$ & $1 \quad(1.0)$ \\
\hline Malaise & $39(38.2)$ & \\
\hline Macular rash & $14(13.7)$ & \\
\hline Fever up & $18(17.6)$ & \\
\hline Dyspnea & $10 \quad(9.8)$ & \\
\hline Peripheral motor neuropathy & $3 \quad(2.9)$ & $1 \quad(1.0)$ \\
\hline Peripheral sensory neuropathy & $27(26.5)$ & $4 \quad(3.9)$ \\
\hline Dysgeusia & $15(14.7)$ & \\
\hline Alopecia & $13(12.7)$ & \\
\hline Pain & $11(10.8)$ & \\
\hline Headache & $5 \quad(4.9)$ & $1 \quad(1.0)$ \\
\hline Hypertension $^{\mathrm{a}}$ & $79(77.5)$ & $31(30.4)$ \\
\hline Epistaxis & $16(15.7)$ & $1 \quad(1.0)$ \\
\hline Thromboembolic event & $7 \quad(6.9)$ & $(4.9)$ \\
\hline Bleeding & $5 \quad(4.9)$ & $1 \quad(1.0)$ \\
\hline Infection & $7 \quad(6.9)$ & $2(2.0)$ \\
\hline Duodenal ulcer & $1 \quad(1.0)$ & $1 \quad(1.0)$ \\
\hline Gait disturbance & $1 \quad(1.0)$ & $1 \quad(1.0)$ \\
\hline Meningitis & $1 \quad(1.0)$ & $1 \quad(1.0)$ \\
\hline
\end{tabular}

${ }^{\mathrm{a}} \mathrm{A}$ total of 47 cases had high blood pressure before bevacizumab treatment. AST, aspartate aminotransferase; ALT, alanine aminotransferase; ALP, alkaline phosphatase.

$(3.9 \%)$. Severe grade 3 or 4 Bev-associated adverse events included hypertension (30.4\%), proteinuria (5.9\%), thromboembolism (4.9\%) and epistaxis (1.0\%). Overall, the majority of adverse events were manageable. No treatment-associated mortality was observed.

Efficacy. The objective response to Bev-containing chemotherapies is summarized in Table III. With 102 evaluable patients, the RR was $44.1 \%$ and the DCR was $92.2 \%$. Only 

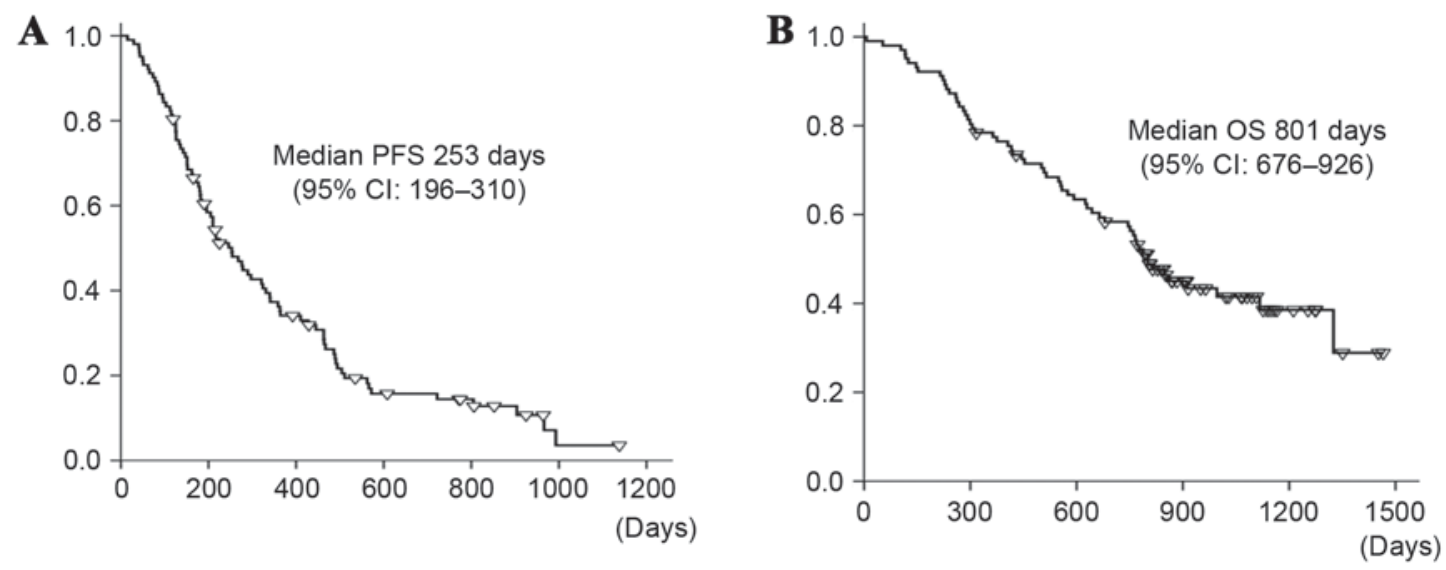

Figure 1. Survival analyses. (A) PFS. (B) OS. PFS, progression-free survival rate; OS, overall survival rate.

Table III. Response to intervention.

\begin{tabular}{lrr}
\hline Response & $\mathrm{n}(\%)$ \\
\hline Complete response & 1 & $(1.0)$ \\
Partial response & 44 & $(43.1)$ \\
Response rate & $(44.1)$ \\
Stable disease & 48 & $(47.1)$ \\
Non-CR/non-PD & 1 & $(1.0)$ \\
Disease control & $(92.2)$ \\
Progressive disease & 5 & $(4.9)$ \\
Not evaluable & 3 & $(2.9)$ \\
\hline
\end{tabular}

1 patient achieved a CR. The median PFS was 8.3 months (95\% CI=6.4-10.2 months), which is longer compared with the predefined expected and threshold values for PFS (6.2 and 4.5 months, respectively), as demonstrated in Fig. 1A. The primary PFS endpoint was met. The median OS was 26.3 months (95\% CI=22.2-30.4 months), as illustrated in Fig. 1B. There was no significant difference in the time to response to BEV between chemotherapy-naïve: Median, 73 days; range, 25-519 days, and previously treated patients: Median, 40 days; range, 23-535 days $(\mathrm{P}=0.265)$. No significant difference was observed in PFS between patients with SD, 268 days $(95 \%$ $\mathrm{CI}=196-416$ days) and those who achieved PR, 266 days (95\% $\mathrm{CI}=125-431$ days) by landmark analysis $(\mathrm{P}=0.97)$. There was also no significant difference observed in OS between patients with SD, 860 days (95\% CI=640-1079 days) and those who achieved PR, 664 days (95\% CI=329-999 days) by landmark analysis $(\mathrm{P}=0.35)$. Univariate and multivariate analyses were performed to identify the variables significantly associated with PFS and OS, as summarized in Tables IV and V. Univariate analysis revealed that several clinical factors were associated with PFS $(\mathrm{P}<0.15)$, as illustrated in Table IV. In the crude model, multivariate analysis indicated that a higher T factor, EGFR exon 21 mutations, and poor PS were linked to significantly shorter PFS. Subsequent to adjustment for pretreatment with EGFR-TKIs, multivariate analysis revealed that a higher $\mathrm{T}$ factor [adjusted hazard ratio $(\mathrm{HR})=1.33$ ] and poor PS (adjusted $H R=1.63$ ) were associated with significantly shorter PFS, whilst EGFR exon 19 deletion (adjusted HR=0.47) was associated with prolonged PFS $(\mathrm{P}<0.05)$, as demonstrated in Table V. In the crude model for OS, multivariate analysis indicated that a lower $\mathrm{T}$ factor and EGFR exon 19 deletion were significant favorable prognostic factors. Subsequent to adjustment for pretreatment with EGFR-TKIs, multivariate analysis revealed that $E G F R$ mutations (adjusted $H R=0.20$ ), better PS (adjusted HR=0.45), and the absence of primary site lesions (adjusted $H R=0.29$ ) were significant favorable prognostic factors $(\mathrm{P}<0.05)$. The Kaplan-Meier estimation of OS was significantly higher for patients with the EGFR exon 19 mutations compared with the estimation for patients with the $E G F R$ exon 21 mutations or wild-type EGFR $(\mathrm{P}=0.037)$.

\section{Discussion}

The present study evaluated the efficacy and safety of Bev-containing chemotherapy regimens in clinical practice in Japan, as first and later lines of chemotherapy. The RR of the present cohort study was $44.1 \%$, and the median PFS was 8.3 months, data that demonstrates better efficacy for patients compared with those of previous reports (E4599, RR 35\%, PFS 6.2 months; ARIES, RR 49\%, PFS 6.6 months) (2,3). The primary endpoint of the present study, PFS, was met, suggesting that Bev-containing chemotherapy is effective in clinical settings in Japan. The median OS, 26.3 months, was also improved compared with those of previous trials (E4599, 12.3 months; ARIES, 13.0 months; SAiL, 14.6 months) (1-3). The effect of Bev was similar to that observed in a Japanese phase II trial that used Bev as first-line chemotherapy (JO19907) (10), which reported an RR of $60.7 \%$, a median PFS of 6.9 months and a median OS of 22.8 months, although the present study included patients receiving first-line and subsequent Bev treatment.

With respect to the severe adverse events of special interest due to Bev treatment, grade III or higher hypertension, proteinuria, thromboembolism and epistaxis occurred in 30.4, $5.9,4.9$ and $1.0 \%$ of patients, respectively, in the present study compared with rates of 7, 3.1, 0.2 and $0.7 \%$, respectively, in the E4599 trial (3) and 6, 3, 8 and 1\%, respectively, in the SAiL study (1). A total of $11 \%$ of patients exhibited severe hypertension in the JO19907 study conducted in Japan (10). The higher incidence of hypertension in the current study may reflect the 
Table IV. Progression-free survival by univariate Cox's regression analyses using an adjusted model for pretreatment of EGFR-TKI $(\mathrm{P}<0.15)$.

\begin{tabular}{lcc}
\hline Variable & HR & 95\% CI \\
\hline Performance status $(0,1,2)$ & 1.94 & $1.26-2.98$ \\
T factor $(0,1,2,3,4)$ & 1.30 & $0.08-1.54$ \\
N factor $(0,1,2,3)$ & 1.25 & $1.05-1.49$ \\
Brain metastasis (no/yes) & 2.22 & $1.16-4.26$ \\
Target lesion (no/yes) & 2.62 & $1.19-5.76$ \\
Recurrence subsequent to surgery vs. IIIA-IV & 2.17 & 0.003 \\
History of surgery (no/yes) & 0.58 & 0.01 \\
Primary site (no/yes) & 2.01 & $0.35-0.93$ \\
EGFR exon 19 deletion (no/yes) & 0.57 & $1.03-3.90$ \\
Combined treatment (taxane/PEM) & 0.61 & $0.33-0.98$ \\
Chemotherapy regimen (PEM/docetaxel/Pt + PEM/Pt + taxane) & 1.28 & $0.38-1.01$ \\
\end{tabular}

TKI, EGFR-tyrosine kinase inhibitor; Taxane, paclitaxel or docetaxel; PEM, pemetrexed; Pt, platinum agent; CI, confidence interval.

Table V. Progression-free survival by multivariate Cox's regression analyses using an adjusted model for pretreatment of EGFR-TKI.

\begin{tabular}{llll}
\hline Variable & HR & 95\% CI & P-value \\
\hline T factor $(0,1,2,3,4)$ & 1.33 & $1.10-1.61$ & 0.003 \\
EGFR exon 19 deletion (no/yes) & 0.47 & $0.25-0.87$ & 0.02 \\
Performance status $(0,1,2,3,4)$ & 1.63 & $1.02-2.60$ & 0.04 \\
N factor $(0,1,2,3)$ & 1.17 & $0.97-1.41$ & 0.11 \\
Combined treatment (taxane/PEM) & 0.73 & $0.41-1.30$ & 0.28 \\
Brain metastasis (no/yes) & 0.87 & $0.39-1.95$ & 0.74 \\
\hline
\end{tabular}

Taxane, paclitaxel or docetaxel; PEM, pemetrexed; CI, confidence interval.

baseline characteristics of the patients, such as the higher incidence of comorbid hypertension at the time of Bev-containing chemotherapy initiation: $46.1 \%$ of patients exhibited hypertension at baseline. Although the present study revealed a higher incidence of hypertension, all toxicity was manageable.

The EGFR mutation type was a predictive marker for Bev-containing chemotherapy efficacy in the present study, with the EGFR exon 19 mutation being a favorable predictor of PFS. Previously, several studies suggested that the effects of EGFR-TKI treatment differ according to the type of EGFR mutation (11-13). Regarding treatment with afatinib, a second-generation irreversible EGFR-TKI, the EGFR mutation type was associated with OS in patients with EGFR mutation-positive lung adenocarcinoma (11). Patients with the EGFR exon 19 deletion experienced a significant survival benefit with afatinib treatment compared to chemotherapy, although no such benefit was observed in patients with the EGFR exon 21 mutations (11). In two phase II studies of EGFR-TKIs, erlotinib or gefitinib, plus Bev, improved PFS was seen upon addition of Bev to the EGFR-TKI regimens in the patients with the EGFR exon 19 deletion, but not for the patients with the exon 21 mutations $(12,13)$. The reason for the Bev-mediated survival benefit in patients with the EGFR exon 19 deletions remains unclear. Several studies have reported distinct biochemical properties of different EGFR mutations that may explain the different responses to EGFR-TKIs $(14,15)$. An association between EGFR and vascular endothelial growth factor (VEGF) has also been reported. EGFR-mutated tumors display higher VEGF expression levels than wild-type EGFR tumors (16). EGFR-TKI-resistant tumors also produce greater levels of VEGF, and the amount of VEGF production varies according to the mutation type $(17,18)$. These data may explain the favorable efficacy of Bev-containing chemotherapy in patients with EGFR exon 19 deletions.

There were several limitations to the present study. Firstly, the sample size was small compared with those in previous studies (SAiL, n=2212; ARIES, n=1967) (1-2). Secondly, the treatment was not restricted to first-line chemotherapy, although Bev-containing chemotherapy was used for first-line treatment. Concerning the OS, the higher frequency of EGFR mutations in the Japanese population may favorably affect patient outcomes compared with previous reports (SAiL and ARIES). In a Japanese phase II study (10), OS was 22.8 months and PFS was 6.9 months for first-line treatment with CBDCA + PTX + Bev. In that trial, the EGFR mutation data were not available, but $41 \%$ of patients received EGFR-TKIs as post-protocol therapy. 
In the present study, EGFR mutations were exhibited in $43.1 \%$ of patients. A considerable percentage of patients may have benefited from post-progression treatment with EGFR-TKIs. A major strength of the present study was that EGFR mutation status data were available for the majority of patients. This allowed the importance of EGFR exon 19 mutation as a possible predictive marker for Bev treatment efficacy to be elucidated.

In conclusion, Bev-containing combination chemotherapy was effective in treating patients with non-sq NSCLC in clinical settings in Japan. Adverse events were well-tolerated and acceptable. Even though these are the results of ad hoc analyses, multivariate analysis revealed that a lower $\mathrm{T}$ factor, better PS, and the EGFR exon 19 mutations were associated with prolonged PFS.

\section{References}

1. Crinò L, Dansin E, Garrido P, Griesinger F, Laskin J, Pavlakis N, Stroiakovski D, Thatcher N, Tsai CM, Wu YL and Zhou C: Safety and efficacy of first-line bevacizumab-based therapy in advanced non-squamous non-small-cell lung cancer (SAiL, MO19390): A phase 4 study. Lancet Oncol 11: 733-740, 2010.

2. Lynch TJ Jr, Spigel DR, Brahmer J, Fischbach N, Garst J, Jahanzeb M, Kumar P, Vidaver RM, Wozniak AJ, Fish S, et al: Safety and effectiveness of bevacizumab-containing treatment for non-small-cell lung cancer: Final results of the ARIES observational cohort study. J Thorac Oncol 9: 1332-1339, 2014.

3. Sandler A, Gray R, Perry MC, Brahmer J, Schiller JH, Dowlati A, Lilenbaum R and Johnson DH: Paclitaxel-carboplatin alone or with bevacizumab for non-small-cell lung cancer. N Engl J Med 355: 2542-2550, 2006.

4. Lara PN Jr, Redman MW, Kelly K, Edelman MJ, Williamson SK, Crowley JJ and Gandara DR; Southwest Oncology Group: Disease control rate at 8 weeks predicts clinical benefit in advanced non-small cell lung cancer: Results from Southwest Oncology Group randomized trials. J Clin Oncol 26: 463-467, 2008.

5. Miyazaki M, Naoki K, Sato T, Tanaka K, Tsuzuki K, Yoshida S, Tomomatsu K, Tasaka S, Soejima K, Sayama K and Asano K: A case of advanced lung adenocarcinoma with cavity formation shrunken by bevacizumab added on the 3rd course of 6th-line chemotherapy. Gan To Kagaku Ryoho 39: 421-424, 2012 (In Japanese).

6. Paez JG, Jänne PA, Lee JC, Tracy S, Greulich H, Gabriel S, Herman P, Kaye FJ, Lindeman N, Boggon TJ, et al: EGFR mutations in lung cancer: Correlation with clinical response to gefitinib therapy. Science 304: 1497-1500, 2004.

7. Naoki K, Soejima K, Okamoto H, Hamamoto J, Hida N, Nakachi I, Yasuda H, Nakayama S, Yoda S, Satomi R, et al: The PCR-invader method (structure-specific 5' nuclease-based method), a sensitive method for detecting EGFR gene mutations in lung cancer specimens; comparison with direct sequencing. Int J Clin Oncol 16: 335-344, 2011.

8. Eisenhauer EA, Therasse P, Bogaerts J, Schwartz LH, Sargent D, Ford R, Dancey J, Arbuck S, Gwyther S, Mooney M, et al: New response evaluation criteria in solid tumours: Revised RECIST guideline (version 1.1). Eur J Cancer 45: 228-247, 2009.
9. http://ctep.cancer.gov/protocolDevelopment/electronic_applications/ctc.htm\#ctc_40

10. Niho S, Kunitoh H, Nokihara H, Horai T, Ichinose Y, Hida T, Yamamoto N, Kawahara M, Shinkai T, Nakagawa K, et al: Randomized phase II study of first-line carboplatin-paclitaxel with or without bevacizumab in Japanese patients with advanced non-squamous non-small-cell lung cancer. Lung Cancer 76: 362-367, 2012

11. Yang JC, Wu YL, Schuler M, Sebastian M, Popat S, Yamamoto N, Zhou C, Hu CP, O'Byrne K, Feng J, et al: Afatinib versus cisplatin-based chemotherapy for EGFR mutation-positive lung adenocarcinoma (LUX-Lung 3 and LUX-Lung 6): Analysis of overall survival data from two randomised, phase 3 trials. Lancet Oncol 16: 141-151, 2015

12. Seto T, Kato T, Nishio M, Goto K, Atagi S, Hosomi Y, Yamamoto N, Hida T, Maemondo M, Nakagawa K, et al: Erlotinib alone or with bevacizumab as first-line therapy in patients with advanced non-squamous non-small-cell lung cancer harbouring EGFR mutations (JO25567): An open-label, randomised, multicentre, phase 2 study. Lancet Oncol 15: 1236-1244, 2014.

13. Ichihara E, Hotta K, Nogami N, Kuyama S, Kishino D, Fujii M, Kozuki T, Tabata M, Harada D, Chikamori K, et al: Phase II trial of gefitinib in combination with bevacizumab as first-line therapy for advanced non-small cell lung cancer with activating EGFR gene mutations: The Okayama Lung Cancer Study Group Trial 1001. J Thorac Oncol 10: 486-491, 2015.

14. Okabe T, Okamoto I, Tamura K, Terashima M, Yoshida T, Satoh T, Takada M, Fukuoka M and Nakagawa K: Differential constitutive activation of the epidermal growth factor receptor in non-small cell lung cancer cells bearing EGFR gene mutation and amplification. Cancer Res 67: 2046-2053, 2007.

15. Zhu JQ, Zhong WZ, Zhang GC, Li R, Zhang XC, Guo AL, Zhang YF, An SJ, Mok TS and Wu YL: Better survival with EGFR exon 19 than exon 21 mutations in gefitinib-treated non-small cell lung cancer patients is due to differential inhibition of downstream signals. Cancer Lett 265: 307-317, 2008.

16. Reinmuth N, Jauch A, Xu EC, Muley T, Granzow M, Hoffmann H, Dienemann H, Herpel E, Schnabel PA, Herth FJ, et al: Correlation of EGFR mutations with chromosomal alterations and expression of EGFR, ErbB3 and VEGF in tumor samples of lung adenocarcinoma patients. Lung Cancer 62: 193-201, 2008.

17. Li H, Takayama K, Wang S, Shiraishi Y, Gotanda K, Harada T, Furuyama K, Iwama E, Ieiri I, Okamoto I and Nakanishi Y: Addition of bevacizumab enhances antitumor activity of erlotinib against non-small cell lung cancer xenografts depending on VEGF expression. Cancer Chemother Pharmacol 74: 1297-1305, 2014.

18. Nakade J, Takeuchi S, Nakagawa T, Ishikawa D, Sano T, Nanjo S, Yamada T, Ebi H, Zhao L, Yasumoto K, et al: Triple inhibition of EGFR, Met, and VEGF suppresses regrowth of HGF-triggered, erlotinib-resistant lung cancer harboring an EGFR mutation. J Thorac Oncol 9: 775-783, 2014. 